\title{
The Raman LIDAR for the pre-production phase of Cherenkov Telescope Array.
}

\author{
Marco Iarlori ${ }^{1, *}$, Ermanno Pietropaolo ${ }^{2}$, Vincenzo Rizi ${ }^{1}$, Carla Aramo ${ }^{3}$, Laura Valore $^{3}$ for CTA Consortium, Valerio \\ Silvestri $^{1}$, Alberto Cirella ${ }^{1}$, Giovanni Dughera ${ }^{4}$, Stefano Galli ${ }^{1}$, and Marco Marengo ${ }^{4}$. \\ ${ }^{1}$ INFN-GSGC L'Aquila and CETEMPS-DSFC, Università degli Studi dell'Aquila, via Vetoio, 67100, L'Aquila, Italy. \\ ${ }^{2}$ INFN-GSGC L'Aquila and DSFC, Università degli Studi dell'Aquila, via Vetoio, 67100, L'Aquila, Italy. \\ ${ }^{3}$ INFN Napoli and Dipartimento di Fisica, Università degli Studi di Napoli "Federico II", Napoli, Italy. \\ ${ }^{4}$ INFN Torino, Italy.
}

\begin{abstract}
The ARCADE Raman lidar has been tested and validated at L'Aquila before the deployment at CTA (Cherenkov Telescope Array) North site. Some modifications have been done on the original ARCADE system to improve its performances, and an extensive programme of measurements has been performed. The Raman lidar (RL) technique is discussed with specific care to the technical constrains of RL systems, and the signal analysis, i.e., the estimation of aerosol optical depth, volume backscatter coefficient, and water vapour profiles and their significance (errors and resolution). Some final comments and conclusions are outlined.
\end{abstract}

\section{Introduction}

The development of the Extensive Air Showers (EAS) generated by gamma primaries is greatly influenced by the atmosphere. The variable atmospheric properties affect the observations of the EAS Cherenkov light in multiple ways being responsible at the same time for the production of the Cherenkov light and also for its attenuation and propagation when it travels towards the telescopes.

The production of Cherenkov light depends on the molecular profile, while the aerosol profile affects its attenuation and propagation; for the details of the propagations, the water vapour profile can be also a useful information [1]. The ARCADE RL is able to provide information about the aerosol properties on site at $355 \mathrm{~nm}$ as well as water vapour profiles. This lidar can be used for the calibration of other instruments that are expected to operate in the future at the CTA site [2].

We briefly describe the ARCADE system details and performances as results of extensive tests made in L'Aquila.

\section{The ARCADE Raman Lidar}

After it operated in Colorado, the ARCADE RL has been moved at L'Aquila to be upgraded for the pre-production phase of CTA (see Fig.1). To achieve this goal some hardware modifications to the system and an intensive campaign of measurements have been performed. Moreover, a number of tools have been developed for the acquisition of the data as well as for their analysis.

\subsection{System details}

Most of the ARCADE RL system characteristics can be found in [2]; below we discuss some of the modifications made in L'Aquila. The hardware changes have been made to get better performances in both the transmitting and receiver section and to setup the water vapour channel. Some improvements in the optical setup have been made after a Zemax ${ }^{\circledR}$ simulation-driven re-design of the combination of the different components [3]. The mechanical setup was not substantially changed, although altazimuth movement automation has been temporary overridden for testing purposes.

The DAQ have been completely redone using new acquisition cards with an in-house developed software.

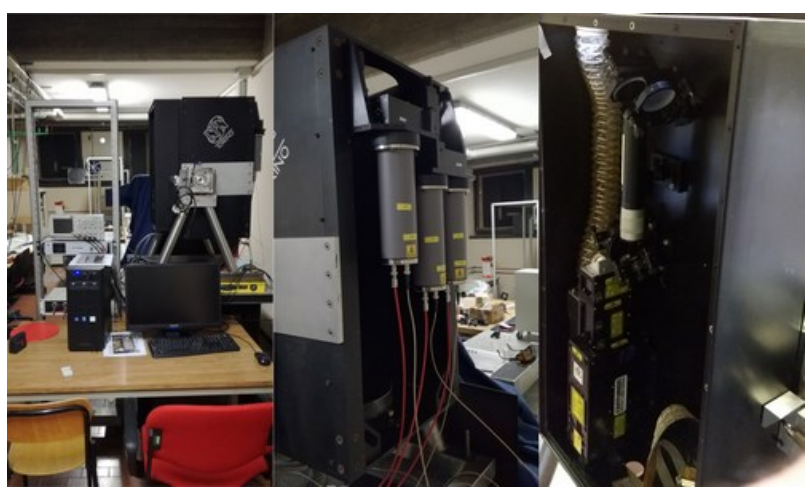

Fig. 1. The ARCADE RL in operational mode at L'Aquila. Left picture: side view. Central picture: detail of the receiver

\footnotetext{
* Corresponding author: marco.iarlori@aquila.infn.it
} 
(telescope \& PMTs). Right picture: detail of the transmitter (laser \& transmitting optics).

\subsubsection{Transmitter section}

The transmitter section is based on a Quantel Centurion $\mathrm{Nd}$ :YAG laser firing@355nm with a repetition rate of $100 \mathrm{~Hz}$. The laser transmitter part increases the beam spectral purity and the reduces the divergence by a factor 10.

The 10x beam expander (BE) and the dichroic beam splitters (DBS) have been substituted by more efficient ones from ThorLabs and Manx Optics respectively (see Fig.2).
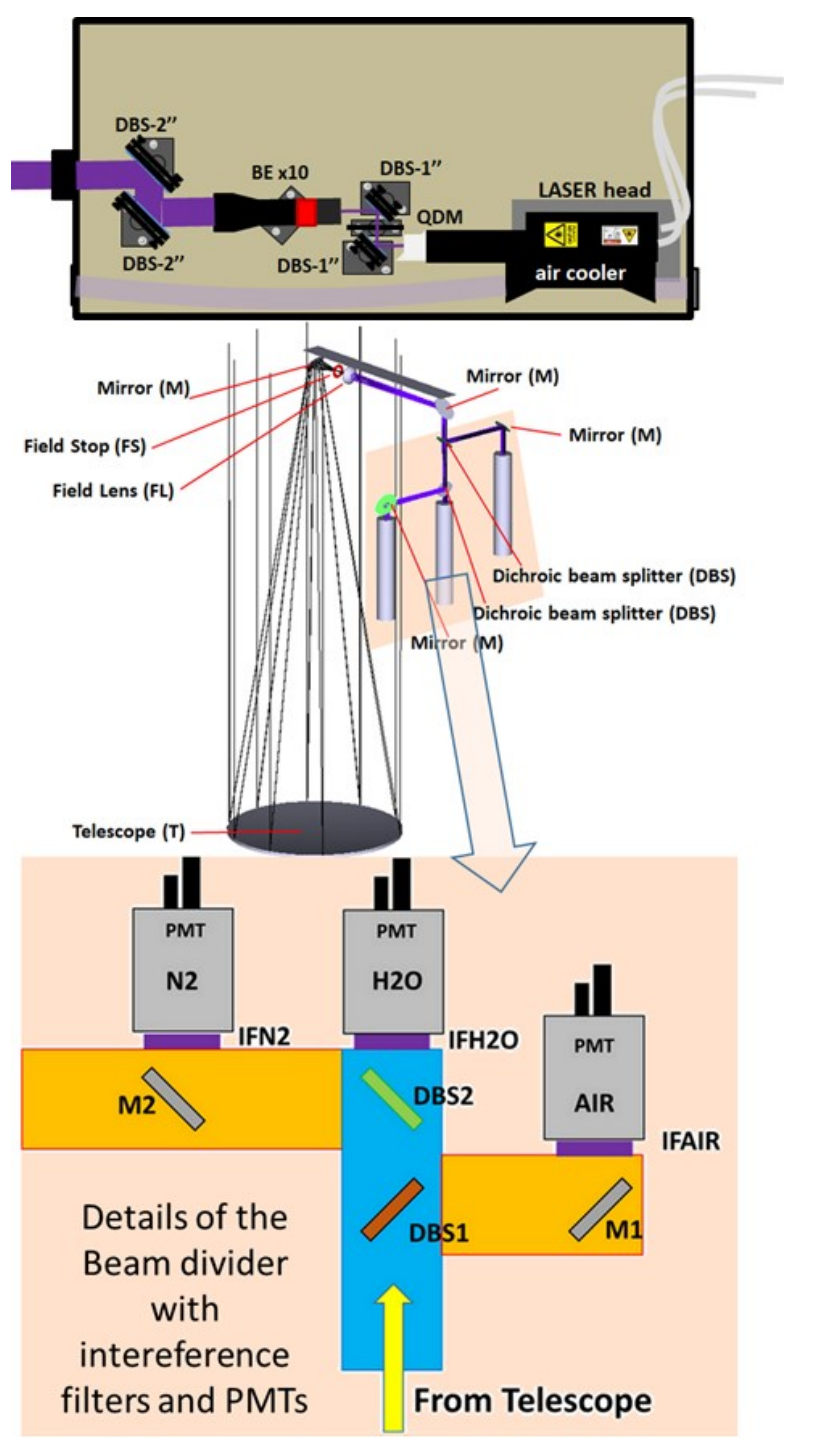

Fig. 2. Upper panel: the layout of the laser transmitter stage; the diameter of the different DBS is indicated (1" or 2"), the purple bar represents the laser beam. Bottom panel: the schematic representation of the receiver, the wavelength separator with DBSs and the acquisition channels with interferential filters (IF) are shown. The mirrors and DBSs are chosen to optimize the wavelength separations.

In the final setting, the laser beam has a spectral purity better than $99.9 \%$, the energy per pulse is about $6 \mathrm{~mJ}$, and the beam half angle divergence is below $0.3 \mathrm{mrad}$. In addition, the heat dissipation of the laser head has been improved to allow long lasting laser firing.

\subsubsection{The Receiver section}

The receiver section is constituted by a $\varnothing 25 \mathrm{~cm} \mathrm{f} / 3$ Marcon parabolic mirror; a combination of mirrors (M) and field lens (FL) sends the collected light to a beam divider that gets the correct wavelength separation between the elastic channel $(355 \mathrm{~nm})$ and the two Raman channels $\left(\mathrm{N}_{2}\right.$ at $387 \mathrm{~nm}$ and $\mathrm{H}_{2} \mathrm{O}$ at $\left.407 \mathrm{~nm}\right)$. In each channel a narrow-band interference filter further enhances the photon selection. The light is detected by a 2" Electron Tubes 9829QSB photomultiplier tube (PMT), the output signal is read by the data acquisition system (APC 2.6 ISOCOMP) in analog and photon-counting ( $\mathrm{PhC})$ acquisition mode. The layout of the receiver section is in Fig.2. The optical efficiencies of each channel can be estimated taking into account the spectral specifications of DBS, IF and the quantum efficiency of the PMTs.

\subsubsection{DAQ control}

The DAQ control is done using in-house Matlab ${ }^{\circledR}$ codes: a software tool with a graphical interface enables to setup the DAQs with the needed parameters (threshold values, FIFO length, etc.), collects the data in both $\mathrm{PhC}$ and analog mode, starts the acquisition and stores the data. The graphical interface is useful, especially during the test phase, but for the operative measurements at ORM, its command line counterpart is more suitable. In operational mode, the DAQ control permits to run the data acquisition with all the necessary automation, for example it enables to:

- open/close the dome;

- control the altazimuth movements;

- $\quad$ read the weather station data;

- $\quad$ power up/starts all the lidar hardware;

- start/stop the acquisition accordingly to the ORM policy;

- $\quad$ shutdown the system in emergency situation;

- process the data analysis.

These software tools are independent from the operative system of the personal computer.

\subsubsection{Transmitter/Receiver test examples}

The transmitter section has been tested to verify the laser pulse characteristics. The Q-switch and diode pulse delay have been set to maximize the energy for a pulse repetition rate of $100 \mathrm{~Hz}$, without degrading the pulse shape. The laser energy and spectral purity have been checked with a spectrometer in critical position along the laser path. In addition, the half-angle beam divergence has been measured: $\theta=0.3 \pm 0.05 \mathrm{mrad}$, which is consistent with the expected result. The beam expander has a fine tuning control that has been exploited to optimize its performances by both measurements and Zemax ${ }^{\circledR}$ simulations. Several tests on the receiver section has been done. Detailed control and setup procedures have been developed, like the one that make use of three laser levels 
to verify the correct alignment of the telescope with the optics of the receiver. The correct alignment between the optical axis of the telescope and laser beam is critical for successful measurements. Zemax ${ }^{\circledR}$ simulations have been used to estimate the displacement from the infinity focus position of the telescope (de-focusing) of the FL to allow the optimal operations in the near field (better overlap) [4], without affecting the far field signals (Fig.3): for ranges $>500 \mathrm{~m}$ the overlap efficiency remains constant (equal to its max value). Tests during actual measurements in atmosphere shown a slight higher value for the full overlap. A maximum overlap efficiency less than unity is expected because the telescope obscuration caused by the holder of the FL and the first two mirrors (M). The spectral efficiencies have been also measured in different positions of light path in the receiver.

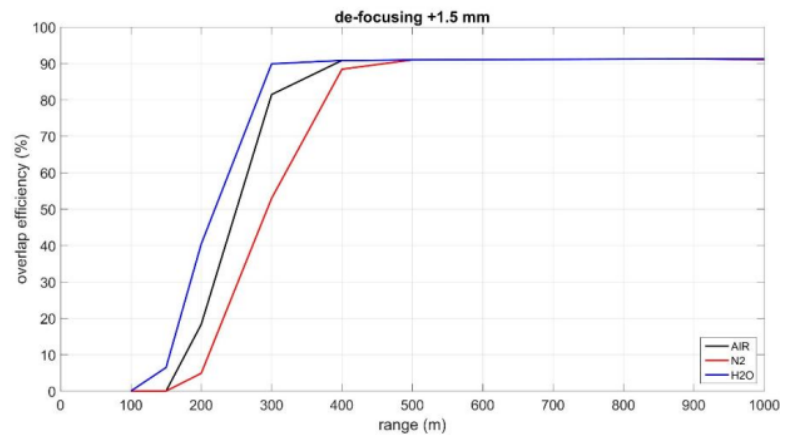

Fig. 3. The overlap efficiency functions of the ARCADE RL estimated with Zemax ${ }^{\circledR}$. The elastic channel (AIR) and the water vapour Raman channel $\left(\mathrm{H}_{2} \mathrm{O}\right)$ reach the max efficiency (the complete overlap) before the nitrogen channel $\left(\mathrm{N}_{2}\right)$ because of the shorter optical path in the receiver.

The PMTs single photons characteristic have been checked to confirm that they are suitable for $\mathrm{PhC}$ measurements, and the best PMT High Voltage (HV) and $\mathrm{PhC}$ threshold level (typically $10 \mathrm{mV}$ ) have been set using their measured pulse height distribution. The specific timing of each acquisition channel, have been checked in the final setup of the DAQ: FIFO length 4096 bins, and dwell time of the single bin at 40ns; this time duration enables to identify the first bin where the actual backscatter signal starts with an error estimated in $\pm 20 \mathrm{~ns}$. This is the so-called "zero-bin" position, an important parameter that has to be determined with enough precision to minimize the VAOD systematic uncertainty [5].

\subsection{Data analysis}

Software tools have been developed to simulate and process the lidar data. Simulation have been used, for example, to estimate the needed attenuation to avoid saturation problems in $\mathrm{PhC}$ mode. The processing of the data is based on the Raman lidar technique to retrieve the relevant aerosol optical properties profiles:

- the aerosol extinction (AE),

- VAOD

- aerosol backscatter (AB), and

- the water vapour (WV) profile.

The elastic signal standard inversion (iterative method) for the retrieval of the $\mathrm{AB}$ only is also used; in the current vertical pointing setup, it is useful mainly for testing. The uncertainties and the effective resolution (ERes) [6] of these data are also evaluated.

\subsubsection{Raw data analysis}

A needed input for RL signal analysis is the local atmospheric molecular density profile. For the measurements taken at L'Aquila we have used a database made with the local rawinsonde observation (RAOB). At ORM, we plan to use the GDAS model or similar data. GDAS data are easily available online and are proven to be of good quality [7]. We could use the GDAS $1^{\circ}$ lat/lon grid data, interpolated on the ORM site, but we are also testing the possibility to use the finer grid of GDAS data at $0.5^{\circ}$ and $0.25^{\circ}$ resolution. Simulated lidar profiles are used vs. the observed ones to exclude the presence of any distortion in the lidar return profiles, usually by comparing them in a region of the atmosphere (generally in the far range) free of aerosols, this is the so called "Rayleigh fit" test. The results of this test on the ARCADE RL signals revealed no significant problems, although a deeper analysis is necessary to accurately assess the impact this normalization procedure on systematic uncertainties on the products retrievals [5].

\subsubsection{Aerosol and water vapour retrievals}

Our codes for the retrieval of the vertical profiles of the aerosol optical properties has been extensively tested $[8,9]$. The details of the aerosol inversions algorithms can be found in [10]. The WV analysis has also been tested in an intercomparison campaign [11]. The ERes and the analysis of the digital filters for lidar signal inversions, can be found in [6].
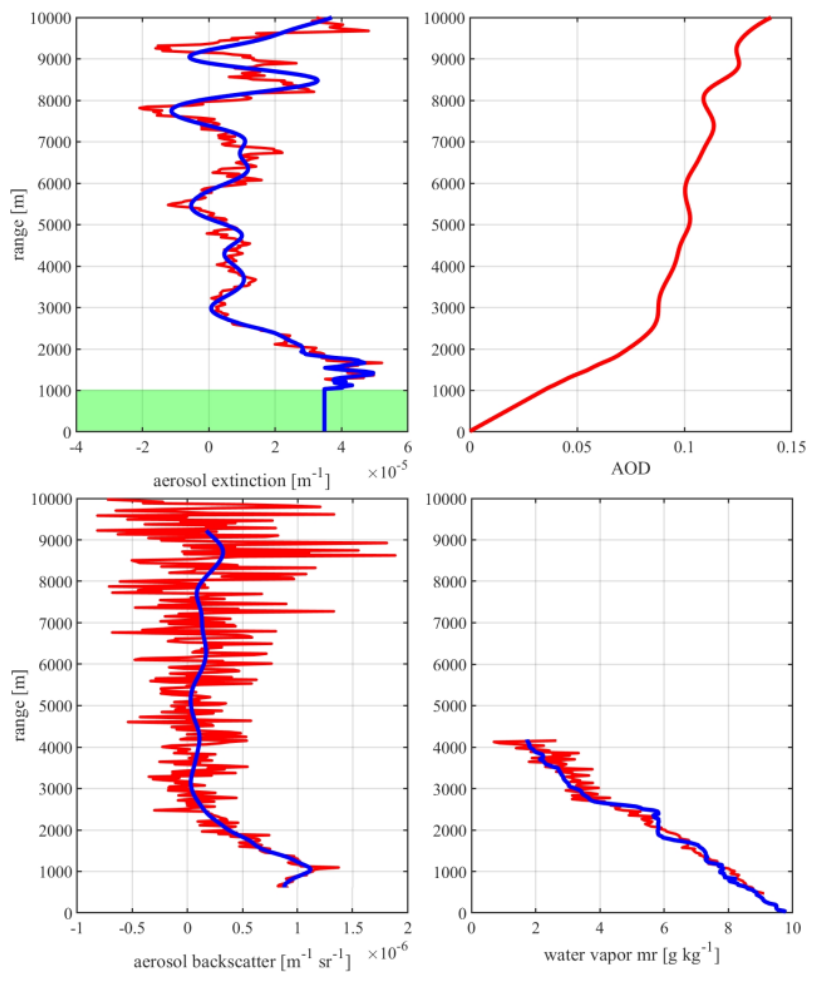
Fig. 4. Upper left panel: below $1 \mathrm{~km}$, there is not a complete optical overlap. The red line is the $\mathrm{AE}$ profile retrieved with a Savitzty-Golay filter; the blue line indicates the AE profile obtained with a Gaussian filter, both profiles have the same ERes. Upper right panel: the AOD profile. Bottom left panel: the $\mathrm{AB}$ profile, in red at raw resolution, in blue at the same $\mathrm{AE}$ ERes. Bottom right panel: the WV profile from the RL data (red) compared with co-located RAOB (blue).

As an example we report some insights concerning a test measurement taken on 15 June 2018. there was a quite low aerosol load in the boundary layer (BL), and the average clear sky AOD was 0.2 , as measured by the co-located sunphotometer, which is part of the AERONET network (data available online at https://aeronet.gsfc.nasa.gov/).

During the whole measurement session we had the occurrence of low level clouds, but the aerosol and the water vapour retrievals in Fig. 4 and are relative to a $15 \mathrm{~min}$ time window with clear sky conditions.

Looking at Fig.4, the AE profile shows a significant aerosol presence up to $2500 \mathrm{~m}$; in this region the $\mathrm{AE}$ has a random error of about $1.5 \times 10^{-5} \mathrm{~m}^{-1}$. Because the good mixing efficiency in the BL, the first good $\mathrm{AE}$ data was taken as the value for ranges where the overlap is not complete. The two AE profiles are relative to retrievals done with different digital filter, with the same ERes: they are shown to point out their different behaviour in removing the high frequency noise.

The VAOD at $3 \mathrm{~km}$ is 0.085 , and the total AOD is about 0.14 , which is compatible with the measurements of the sunphotometer. Different AOD evaluation methods evidenced a possible systematic that should be further investigated. The overall systematic error is assessed of the order of \pm 0.01 , while the random error can be estimated of about 0.003 .

The $\mathrm{AB}$ is plotted at raw resolution $(30 \mathrm{~m})$ as well as at the same ERes of the AE, the latter one has a reduced random error (up to $0.5 \times 10^{-7} \mathrm{~m}^{-1} \mathrm{sr}^{-1}$ in the $\mathrm{BL}$ ) and enables to calculate the lidar ratio, a parameter that contains information about the aerosol particle dimension and composition.

The ERes is variable (from $300 \mathrm{~m}$ to $1000 \mathrm{~m}$ ) to deal with the SNR level of the lidar returns; the ERes can be optimized, for example, to achieve a certain random error level, to limit the distortions in the profile, etc. The WV mixing ratio profile is also shown in Fig. 4, and exhibits a good agreement with the nearly co-located RAOB. The maximum altitude of this profile is only $4000 \mathrm{~m}$ due to several reasons, the main one is a low SNR for the $\mathrm{H}_{2} \mathrm{O}$ Raman signal level.

\section{Conclusions}

The ARCADE RL has been partially upgraded and extensively tested in L'Aquila. The overall optical performances have been significantly improved. A number of practical standard procedure have been developed to setup the system for automatic measurements once in ORM.

An efficient DAQ control software and a certified lidar signals inversion code have been also developed in house: both have been tested during several measurement sessions.

We think that a further optimization of the system in both the hardware and software section are possible in order to reduce the uncertainties.

We expect that the system will be operative, i.e., taking preliminary routine measurements in automatic mode before the sunset and just after the sunrise, from the second week of October 2018.

In summary, the RL will provide the vertical profile of:

- the aerosol optical depth (VAOD);

- the aerosol backscatter coefficient (AB);

. the water vapour mixing ratio (WV).

Along 15 minutes data accumulation, the errors (systematic and uncorrelated) of these profiles are, in the altitude range between 0 and $3 \mathrm{~km}$ agl:

$-<0.015$ for VAOD;

$-<0.1 \times 10^{-6} \mathrm{~m}^{-1} \mathrm{sr}^{-1}$ for the aerosol backscatter coefficient; $-<0.5 \mathrm{~g}$ of $\mathrm{H}_{2} \mathrm{O}$ per $\mathrm{kg}$ of air for the water vapour mixing ratio.

This precision is quite enough for the full characterization of the aerosol optical properties and water vapour over the CTA North site at ORM.

After a detailed evaluation of the possible interference with other experiments operating at ORM, we could also organize another schedule of the measurements.

\section{References}

1. K. Bernlöhr, Astropart. Phys., 12, 255-268 (2000).

2. L. Valore, et al., Proc. $34^{\text {th }}$ ICRC (2015) arXiv:1509.02681 (2015).

3. V. Silvestri. Bachelor thesis, University of L'Aquila, Italy (2017).

4. L. Wiencke, et al., J. Instrum., 12(03), P03008 (2017).

5. V. Freudenthaler, et al., Atmos. Meas. Tech. Discuss., https://doi.org/10.5194/amt-2017-395, in review, 2018.

6. M. Iarlori, et al., Atmos. Meas. Tech., 8, 5157-5176, doi: 10.5194/amt-8-5157-2015 (2015).

7. M. Will et al., Astropart. Phys. 35, 591-607 (2012).

8. C. Böckmann, et al., Appl. Opt. 43, 977-989 (2004).

9. G. Pappalardo, et al., Appl. Opt., 43, 5370-5385 (2004).

10. I. Mattis, et al., Atmos. Meas. Tech., 9, 3009-3029, https://doi.org/10.5194/amt-9-3009-2016, (2016).

11. P. D'Aulerio, et al., Reviewed and revised papers presented at the 22nd International Laser Radar Conference, Pappalardo G., Amodeo A.(eds.) ESA SP-561. (2004). 